Pacific Journal of Mathematic 


\title{
ON THE PROJECTIONS OF A CONVEX POLYTOPE
}

\section{ROLF SCHNEIDER}

\begin{abstract}
It is shown that in the class of all centrally symmetric convex bodies in $E^{d}$ a polytope is uniquely determined, up to a translation, by its brightness (or certain similar functionals) in a suitable, though "arbitrarily small", set of directions.
\end{abstract}

It is well known that a centrally symmetric convex body (compact, convex set with interior points) in $d$-dimensional Euclidean space $E^{d}(d \geqq 3)$ is, up to a translation, uniquely determined by its brightness function. To formulate a more general result, let $S^{d-1}:=\left\{x \in E^{d}:\|x\|=1\right\}$ be the unit sphere in $E^{d}$; for a convex body $K \subset E^{d}$ and a unit vector $u \in S^{d-1}$ let $K(u)$ be the convex set that arises by orthogonal projection of $K$ on to the $(d-1)$-dimensional linear subspace orthogonal to $u$. For $p \in\{0,1, \cdots, d-2\}$ let $v_{p}(K, u)$ denote the $p$-th cross-section measure (Quermassintegral; for a definition see Bonnesen-Fenchel [2, p. 49], or Hadwiger [5, p. 209]) of dimension $d-1$ of the set $K(u)$. Thus, e.g., $v_{0}(K, u)$ is the brightness of $K$ in the direction $u$, and $v_{d-2}(K, u)$ is, up to a factor depending only on $d$, the mean width of $K(u)$. The following theorem has been proved by A. D. Aleksandrov [1]:

If $K, \bar{K} \subset E^{d}$ are centrally symmetric convex bodies satisfying $v_{p}(K, u)=v_{p}(\bar{K}, u)$ for each $u \in S^{d-1}$ and for some $p \in\{0,1, \cdots, d-2\}$, then $\bar{K}$ is a translate of $K$.

For another proof and a generalization see Chakerian [3].

One might ask whether in Aleksandrov's theorem it is really necessary to assume the equality $v_{p}(K, u)=v_{p}(\bar{K}, u)$ for the set of all directions $u$ or whether some nondense subset thereof might suffice. The latter is, however, not true in general. In fact, given a centrally symmetric convex body $K \subset E^{d}$ with sufficiently smooth boundary and a symmetric subset $A \subset S^{d-1}$ which is not dense in $S^{d-1}$, there exists a centrally symmetric convex body $\bar{K} \subset E^{d}$, not a translate of $K$, which satisfies $v_{0}(K, u)=v_{0}(\bar{K}, u)$ for each $u \in A$. Examples to this effect have been constructed in [7, §4]. The object of the present note is to exhibit a contrary situation: In case $K$ is a centrally symmetric polytope, there exist sets $A \subset S^{d-1}$ of arbitrarily small (positive) measure such that the assumption

$$
v_{p}(K, u)=v_{p}(\bar{K}, u) \text { for each } u \in A
$$


forces the centrally symmetric convex body $\bar{K}$ to be a translate of $K$. More precisely, we shall prove the following

THEOREM. Let $K \subset E^{d}$ be a centrally symmetric convex polytope. Let $p \in\{0,1, \cdots, d-2\}$, and let $A \subset S^{d-1}$ be an open set which contains, corresponding to each $(d-1-p)$-dimensional face of $K, a$ vector which is parallel to that face. If $\bar{K} \subset E^{d}$ is a centrally symmetric convex body which satisfies

$$
v_{p}(K, u)=v_{p}(\bar{K}, u) \quad \text { for each } u \in A,
$$

then $\bar{K}$ is a translate of $K$.

For $p \leqq d-3$ there exist universal sets $A$ with the properties demanded in the theorem. For instance, if $A$ is a neighborhood of an "equator sphere" of $S^{d-1}$, then $A$ contains, corresponding to any $(d-1-p)$-face $F$ of any convex polytope, a vector which is parallel to $F$.

The following remarks are preparatory to the proof of the theorem. For a convex body $K \subset E^{d}$ let $\mu_{p}(K, \cdot), p=1, \cdots, d-1$, be its $p$-th surface area function; thus $\mu_{p}$ is a positive Borel measure on $S^{d-1}$ which may be characterized by the fact that

$$
V(\bar{K}, \underbrace{K, \cdots, K}_{p}, \underbrace{B, \cdots, B)}_{d-1-p}=\frac{1}{d} \int_{s^{d-1}} \bar{h}(v) \mu_{p}(K, d v)
$$

for every convex body $\bar{K} \subset E^{d}$ (see Fenchel-Jessen [4]); here the left side is a mixed volume, $B$ is the ball bounded by $S^{d-1}$, and $\vec{h}$ is the support function of $\bar{K}$. As a special case of (1) we have the representation

$$
v_{p}(K, u)=\frac{1}{2} \int_{S^{d-1}}|\langle u, v\rangle| \mu_{d-1-p}(K, d v), \quad u \in S^{d-1} .
$$

For a convex polytope $P \subset E^{d}$ and $p \in\{1, \cdots, d-1\}$ let $\sigma_{p}(P) \subset S^{d-1}$ be the spherical image of the $p$-faces of $P$, thus, by definition, $u \in \sigma_{p}(P)$ if and only if the supporting hyperplane of $P$ with exterior normal vector $u$ contains a $p$-face of $P$. We assert that the measure $\mu_{p}(P, \cdot)$ is concentrated on $\sigma_{p}(P)$. In fact, if $\omega \in S^{d-1}$ is a Borel set having empty intersection with $\sigma_{p}(P)$, then $\mu_{p}(P, \omega)=0$ as may be seen from the last formula of Fenchel-Jessen [4] and an easy estimate of the measure of the "brush set" corresponding to $\omega$.

We shall need two lemmas concerning expressions of the type occurring in (2). Let $\mu$ be a positive Borel measure on $S^{d-1}$ which is 
symmetric (i.e., attains the same value at antipodal sets). Then

$$
H(x):=\int_{S^{d-1}}|\langle x, v\rangle| \mu(d v)
$$

is, for $x \in E^{d}$, a (symmetric) convex function. Let $H^{\prime}(x ; y)$ for $y \in E^{d} \backslash\{0\}$ denote the directional derivative (see Bonnesen-Fenchel [2, p. 19]) of $H$ at $x$ in the direction $y$.

LEMMA 1. If $H$ is given by (3) with symmetric $\mu$, then

where

$$
H^{\prime}(x ; y)=2 \int_{s_{x}}\langle y, v\rangle \mu(d v)+\int_{\omega_{x}}\langle y, v\rangle \mid \mu(d v)
$$

$$
\begin{aligned}
& S_{x}:=\left\{v \in S^{d-1}:\langle x, v\rangle>0\right\}, \\
& \omega_{x}:=\left\{v \in S^{d-1}:\langle x, v\rangle=0\right\} .
\end{aligned}
$$

For the easy computation, see [6, Lemma 6.1].

LeMma 2. If $\mu$ is a symmetric signed Borel measure on $S^{d-1}$ which satisfies

$$
\int_{S^{d-1}}|\langle u, v\rangle| \mu(d v)=0 \text { for each } u \in S^{d-1},
$$

then $\mu=0$.

Essentially, this has been proved by Aleksandrov [1, §8]. In proving his theorem quoted in the introduction, he showed the assertion of Lemma 2 to be true in the case where $\mu$ is a difference of two $(d-1-p)$-th surface area functions of convex bodies; but this assumption is not needed in the proof. To be sure, this is not a special case, since from the well known existence theorem of Minkowski, Aleksandrov, and Fenchel-Jessen [4, p. 16], it follows that every symmetric Borel measure on $S^{d-1}$ is the difference of the $(d-1)$-st surface area functions of two appropriate centrally symmetric convex bodies; hence Lemma 2 follows also directly from Aleksandrov's theorem cited earlier. For further references and a generalization of Lemma 2, see [6].

We proceed now to the proof of the theorem. It is convenient to write $d-1-p=q$. The assumptions of the theorem together with formula (2) give

$$
\int_{S^{d-1}}|\langle u, v\rangle| \mu_{q}(K, d v)=\int_{S^{d-1}}|\langle u, v\rangle| \mu_{q}(\bar{K}, d v)
$$


for each $u \in A$. Let $F$ be a $q$-dimensional face of the polytope $K$. We have assumed that the set $A$ contains a vector $f$ which is parallel to $F$. Since $A$ is an open set it contains a neighborhood of $f$. If equation (4) holds for a unit vector $u$, it holds also for every $\alpha u$, $\alpha>0$; thus there is an open set $U$ of $E^{d}$ containing $f$ such that (4) holds for each $u \in U$. Therefore the convex functions which are defined by the left and the right side of (4), respectively, must have equal directional derivatives at $f$ with respect to every direction $y$. Then Lemma 1 yields

$$
\begin{aligned}
& 2 \int_{S_{f}}\langle y, v\rangle \mu_{q}(K, d v)+\int_{\omega_{f}}|\langle y, v\rangle| \mu_{q}(K, d v) \\
& \quad=2 \int_{S_{f}}\langle y, v\rangle \mu_{q}(\bar{K}, d v)+\int_{\omega_{f}}|\langle y, v\rangle| \mu_{q}(\bar{K}, d v)
\end{aligned}
$$

for each $y \in E^{d}$. If we replace $y$ by $-y$ and add the resulting equation to the former one we see that

$$
\int_{\omega_{f}}|\langle y, v\rangle| \mu_{q}(K, d v)=\int_{\omega_{f}}|\langle y, v\rangle| \mu_{q}(\bar{K}, d v) .
$$

Since $K$ and $\bar{K}$ are centrally symmetric, the measures $\mu_{q}(K, \cdot)$ and $\mu_{q}(\bar{K}, \cdot)$ are symmetric. We can now apply Lemma 2 with the dimension $d$ replaced by $d-1$, with $S^{d-1}$ replaced by $\omega_{f}$, and with $\mu$ replaced by the restriction of $\mu_{q}(K, \cdot)-\mu_{q}(\bar{K}, \cdot)$ to $\omega_{f}$. We deduce that

$$
\mu_{q}\left(K, \omega \cap \omega_{f}\right)=\mu_{q}\left(\bar{K}, \omega \cap \omega_{f}\right)
$$

for every Borel set $\omega$ of $S^{d-1}$. Now observe that the vector $f$ has been chosen parallel to the $q$-face $F$. Thus every unit vector which is orthogonal to $F$ is contained in $\omega_{f}$, hence $\omega_{f}$ contains the spherical image of the face $F$. Therefore equation (6) is especially true if $\omega_{f}$ is replaced by the spherical image of $F$. Now $F$ is an arbitrary $q$-face of $K$, hence the the additivity of the measures allows us to further replace the spherical image of $F$ by the union of the spherical images of the $q$-faces of $K$ :

$$
\mu_{q}\left(K, \omega \cap \sigma_{q}(K)\right)=\mu_{q}\left(\bar{K}, \omega \cap \sigma_{q}(K)\right) .
$$

It has already been noticed that the measure $\mu_{q}(K, \cdot)$ is concentrated on $\sigma_{q}(K)$, therefore to intersect $\omega$ with $\sigma_{q}(K)$ on the left side of (7) is indeed superfluous; we have

$$
\mu_{q}(K, \omega)=\mu_{q}\left(\bar{K}, \omega \cap \sigma_{q}(K)\right)
$$

for every Borel set $\omega$ on $S^{d-1}$. Write 


$$
\nu(\omega):=\mu_{q}(\bar{K}, \omega)-\mu_{q}(K, \omega)
$$

then (8) gives

$$
\nu(\omega)=\mu_{q}\left(\bar{K}, \omega \cap\left[S^{d-1} \backslash \sigma_{q}(K)\right]\right)
$$

so that $\nu$ is still a positive measure. Hence the function

$$
H(x):=\int_{S^{d-1}}|\langle x, v\rangle| \nu(d v),
$$

defined for $x \in E^{d}$, is the support function of a compact convex set $C$. By (4) we have $H(u)=0$ for each $u \in A$, where $A$ is an open set on $S^{d-1}$, and since $H$ is even, we have also $H(u)=0$ for each $u$ in the set antipodal to $A$. Thus $C$ cannot contain a point different from 0 . This gives $H(x)=0$ for each $x \in E^{d}$, and another application of Lemma 2 shows that $\nu$, being symmetric, must vanish identically. We have proved that the convex bodies $K$ and $\bar{K}$ have the same $q$-th surface area function, hence they differ at most by a translation (Aleksandrov [1], Fenchel-Jessen [4]).

\section{REFERENCES}

1. A. D. Aleksandrov, Zur Theorie der gemischten Volumina von konvexen Körpern, II (Russian), Mat. Sbornik N.S. 2 (1937), 1205-1238.

2. T. Bonnesen and W. Fenchel, Theorie der konvexen Körper, Springer, Berlin, 1934.

3. G. D. Chakerian, Sets of constant relative width and constant relative brightness, Trans. Amer. Math. Soc. 129 (1967), 26-37.

4. W. Fenchel and B. Jessen, Mengenfunktionen und konvexe Körper, Danske Vid. Selsk. Mat.-Fys. Medd. 16 (1938).

5. H. Hadwiger, Vorlesungen über Inhalt, Oberfläche und Isoperimetrie, Springer, Berlin-Göttingen-Heidelberg, 1957.

6. R. Schneider, Über eine Integralgleichung in der Theorie der konvexen Körper, Math. Nachr. (to appear).

7. R. Schneider and W. Weil, Über die Bestimmung eines konvexen Körpers durch die Inhalte seiner Projektionen (to appear).

Received July 14, 1969.

MATHEMATISChES INSTITUT DER RUHR-UNIVERSität

463 Bochum, Germany 



\title{
PACIFIC JOURNAL OF MATHEMATICS
}

\author{
EDITORS
}

H. SAMELSON

Stanford University

Stanford, California 94305

J. DugunduI

Department of Mathematics

University of Southern California

Los Angeles, California 90007

RICHARD PIERCE

University of Washington

BASIL GORDON*

University of California

Seattle, Washington 98105

Los Angeles, California 90024

\section{ASSOCIATE EDITORS}

E. F. BeCKenbaCh

B. H. NeumanN

F. WOLF

K. YosHIDA

\section{SUPPORTING INSTITUTIONS}

\author{
UNIVERSITY OF BRITISH COLUMBIA \\ CALIFORNIA INSTITUTE OF TECHNOLOGY \\ UNIVERSITY OF CALIFORNIA \\ MONTANA STATE UNIVERSITY \\ UNIVERSITY OF NEVADA \\ NEW MEXICO STATE UNIVERSITY \\ OREGON STATE UNIVERSITY \\ UNIVERSITY OF OREGON \\ OSAKA UNIVERSITY \\ UNIVERSITY OF SOUTHERN CALIFORNIA
}

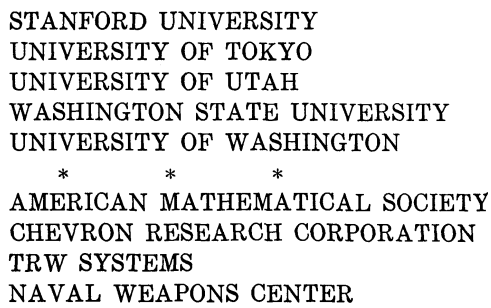

The Supporting Institutions listed above contribute to the cost of publication of this Journal, but they are not owners or publishers and have no responsibility for its content or policies.

Mathematical papers intended for publication in the Pacific Journal of Mathematics should be in typed form or offset-reproduced, double spaced with large margins. Underline Greek letters in red, German in green, and script in blue. The first paragraph or two must be capable of being used separately as a synopsis of the entire paper. It should not contain references to the bibliography. Manuscripts, in duplicate if possible, may be sent to any one of the four editors. Please classify according to the scheme of Math. Rev. 36, 1539-1546. All other communications to the editors should be addressed to the managing editor, Richard Arens, University of California, Los Angeles, California, 90024.

50 reprints are provided free for each article; additional copies may be obtained at cost in multiples of 50 .

The Pacific Journal of Mathematics is published monthly. Effective with Volume 16 the price per volume (3 numbers) is $\$ 8.00$; single issues, $\$ 3.00$. Special price for current issues to individual faculty members of supporting institutions and to individual members of the American Mathematical Society: $\$ 4.00$ per volume; single issues $\$ 1.50$. Back numbers are available.

Subscriptions, orders for back numbers, and changes of address should be sent to Pacific Journal of Mathematics, 103 Highland Boulevard, Berkeley, California, 94708.

PUBLISHED BY PACIFIC JOURNAL OF MATHEMATICS, A NON-PROFIT CORPORATION

Printed at Kokusai Bunken Insatsusha (International Academic Printing Co., Ltd.), 7-17, Fujimi 2-chome, Chiyoda-ku, Tokyo, Japan.

* Acting Managing Editor. 


\section{Pacific Journal of Mathematics}

\section{Vol. 32, No. 3 \\ March, 1970}

Shair Ahmad, Dynamical systems of characteristic $0^{+} \ldots \ldots \ldots \ldots \ldots$

Charles A. Akemann and Bernard Russo, Geometry of the unit sphere of a $C^{*}$-algebra and its dual............................ 575

Philip Bacon, The compactness of countably compact spaces ......... 587

Richard Blaine Barrar and Henry Loeb, On the continuity of the nonlinear Tschebyscheff operator ............................ 593

L. Carlitz, Factorization of a special polynomial over a finite field ....... 603

Joe Ebeling Cude, Compact integral domains .................... 615

Frank Rimi DeMeyer, On automorphisms of separable algebras. II . . . . . 621

James B. Derr, Generalized Sylow tower groups .................. 633

Raouf Doss, Some inclusions in multipliers ................... 643

Mary Rodriguez Embry, The numerical range of an operator........... 647

John Froese, Domain-perturbed problems for ordinary linear differential

operators..................................... 651

Zdeněk Frolík, Absolute Borel and Souslin sets ..................... 663

Ronald Owen Fulp, Tensor and torsion products of semigroups .......... 685

George Grätzer and J. Płonka, On the number of polynomials of an

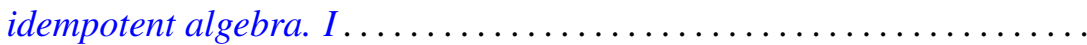

Newcomb Greenleaf and Walter Read, Positive holomorphic differentials on

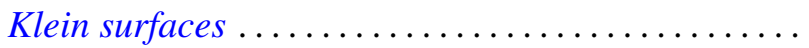

John Willard Heidel, Uniqueness, continuation, and nonoscillation for a second order nonlinear differential equation ................. 715

Leon A. Henkin, Extending Boolean operations................... 723

R. Hirshon, On hopfian groups .......................... 753

Melvin Hochster, Totally integrally closed rings and extremal spaces ..... 767

R. Mohanty and B. K. Ray, On the convergence of a trigonometric integral ..................................

Michael Rich, On a class of nodal algebras .................... 787

Emile B. Roth, Conjugate space representations of Banach spaces ........ 793

Rolf Schneider, On the projections of a convex polytope .............. 799

Bertram Manuel Schreiber, On the coset ring and strong Ditkin sets ...... 805

Edgar Lee Stout, Some remarks on varieties in polydiscs and bounded

holomorphic functions .........................

James Edward Ward, Two-groups and Jordan algebras . 